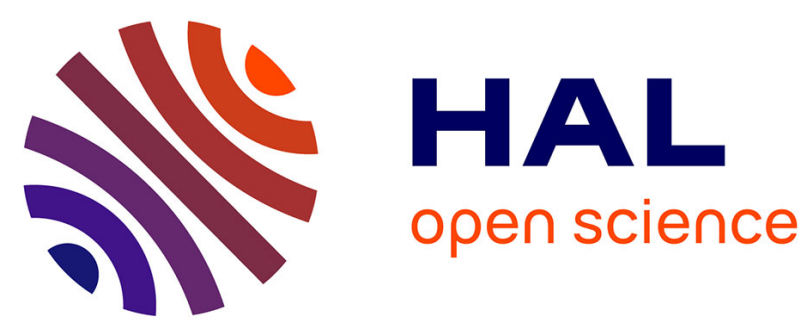

\title{
Characterization of a non linear fractional model of electrode-tissue impedance for neuronal stimulation
}

Florian Kolbl, Jocelyn Sabatier, Gilles N'Kaoua, Frédéric Naudet, Emilie Faggiani, Abdelhamid Benazzouz, Sylvie Renaud, Noëlle Lewis

\section{- To cite this version:}

Florian Kolbl, Jocelyn Sabatier, Gilles N'Kaoua, Frédéric Naudet, Emilie Faggiani, et al.. Characterization of a non linear fractional model of electrode-tissue impedance for neuronal stimulation. Biomedical Circuits and Systems Conference (BioCAS), 2013 IEEE, Nov 2013, Rotterdam, Netherlands. pp.338-341, 10.1109/BioCAS.2013.6679708 . hal-00986311

\section{HAL Id: hal-00986311 \\ https://hal.science/hal-00986311}

Submitted on 2 May 2014

HAL is a multi-disciplinary open access archive for the deposit and dissemination of scientific research documents, whether they are published or not. The documents may come from teaching and research institutions in France or abroad, or from public or private research centers.
L'archive ouverte pluridisciplinaire HAL, est destinée au dépôt et à la diffusion de documents scientifiques de niveau recherche, publiés ou non, émanant des établissements d'enseignement et de recherche français ou étrangers, des laboratoires publics ou privés. 


\title{
Characterization of a Non Linear Fractional Model of Electrode-Tissue Impedance for Neuronal Stimulation
}

\author{
F. Kölbl*, J. Sabatier*, G. N’Kaoua*, F. Naudet ${ }^{\dagger}$, E. Faggiani ${ }^{\dagger}$, A. Benazzouz ${ }^{\dagger}$, S. Renaud* $^{*}$ and N. Lewis* \\ *IMS, UMR 5218 CNRS - University of Bordeaux \\ 351, Cours de la Libération 33405 TALENCE - FRANCE, \\ Email: florian.kolbl@ims-bordeaux.fr \\ ${ }^{\dagger}$ IMN, UMR 5293 CNRS - University of Bordeaux \\ Email: abdelhamid.benazzouz@u-bordeaux2.fr
}

\begin{abstract}
The design of neuro-stimulators must include a realistic model of electrode-tissue interface. Complex electrochemical phenomena associated to high levels of stimulation current give fractional and non linear behavior to this interface that simple linearized models fail to fit. This paper describes both a measurement protocol based on biphasic current-controlled solicitations and a modeling procedure relying on an original approach of multi-model, taking into account the non-linear and fractional effects. This model fits correctly the measurement results with current levels varying from $50 \mu A$ to $1 m A$. Furthermore the whole characterization protocol can be safely transposed to in vivo measurements.
\end{abstract}

\section{ELECTRODE/TISSUE ELECTRICAL MODELING FOR NEURONAL INTERFACE SYSTEMS}

Neuro-electronic systems are based on electrical interaction with neuronal tissue through electrodes. Such an interface allows both recording and stimulating cells. A large variety of extra-cellular electrodes have been developed for in vivo or in vitro applications, with different properties in terms of material and sizing. If large-scale Multi-Electrode-Arrays and macroelectrodes used in Functional Electrical Stimulation or Deep Brain Stimulation (DBS) are indeed very different, the charge transport phenomena at the electrode-electrolyte interface is similar.

The electrical modeling of this interface has been widely addressed in the literature, leading to equivalent models wellknown by specialists in electro-chemistry, theoretical physics or biosensors [1]. The electrical behavior of this interface is complex and integrates fractal and non-linear effects [2][4]. Some authors propose an implementation of the model, compatible with classical electrical simulators [3], [4]. In these approaches, the electrical model is used as a support to extract qualitative information about the tissue or interface properties.

For the design of front-end circuits in neuro-electronic systems, it is important to account for the interface electrical model, because its impedance clearly affects the recorded biopotential and the effective injected current for the stimulation case. When used by circuit designers, the electrical model of the tissue-electrode interface is often over-simplified and linearized, to be easily integrated in electrical simulators
[5], [6]. If this approach is relevant in the recording conditions which correspond to low level voltages across the interface (smaller than $1 \mathrm{mV}$ ), it is no longer acceptable for electrical stimulation where high levels of current are involved. Another important point is the electrical characterization method for the interface. A classical technique is the impedance spectroscopy which assumes the small signal AC behavior.

Our objective is to develop a characterization and modeling protocol of the electrode-tissue interface, in conditions of electrical stimulation and compatible with in vivo measurements. To respect safety conditions and avoid irreversible degradation of the tissue, it is recommended to stimulate in current mode and to use biphasic current impulses. We strictly respect these conditions regarding the input signals used in the characterization protocol. The amplitude of the input waveform is progressively increased to characterize the non-linear behavior and the modeling protocol is also based on these in vivo-compatible signals. As a case study, we consider the experimental investigation of DBS effects on small rodents.

In this paper we describe the whole characterization and modeling procedure, taking into account the fractional and non-linear aspects of the model. We assess the procedure and present results on in vitro measurements, with a standard macro-electrode used for Sub-Thalamic Nucleus stimulation of small rodents.

\section{MEASUREMENT SETUP AND RESUlTS}

Electrical stimulation of biological tissues is preferably done by a current source [7], [8], as it is a natural way to control the injected electrical charge. Balancing the total injected charge is important to avoid tissue damage [7]. In order to reach these specifications, we have developed a specific setup.

\section{A. Impedance measurement with current amplitude control}

The measurement bench uses of a programmable arbitrary waveform generator (Agilent 33250A) to control the stimulus applied to the electrode. This generator has a voltage output, nevertheless our goal is to stimulate the tissue in current mode. For this purpose we designed a conversion circuit shown in figure 1(a). Voltage accross the electrode is sensed by a 


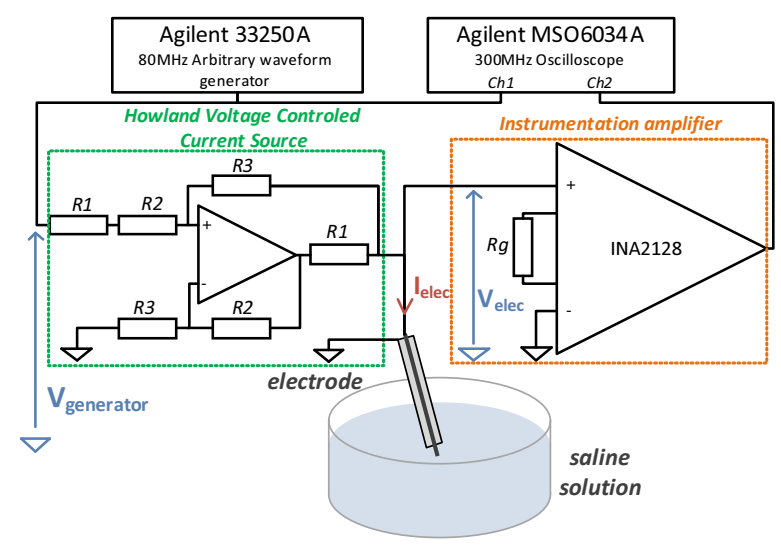

(a)

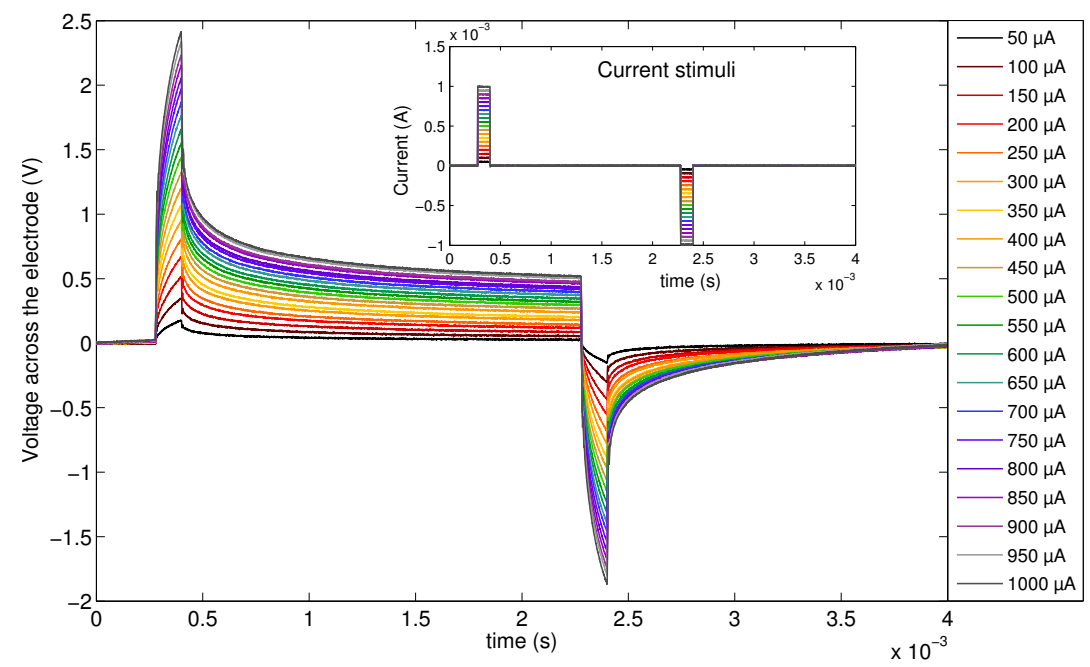

(b)

Fig. 1. 1(a) Impedance measurement system. The spectral analyzer is connected to a Howland Voltage Controlled Current Source (VCCS) and the to electrode; voltage across the electrode $V_{\text {elec }}$ is recorded through an instrumentation amplifier, which gain can vary from 0 to $100 \mathrm{~dB}$. 1(b) Measured voltage step responses for different amplitude of constant current biphasic stimulus.

preamplifier and recorded with an Agilent MSO6034A 300 $\mathrm{MHz}$ Oscilloscope.

Assuming that the input impedance of the instrumentation amplifier is infinite, the Howland Voltage Controlled Current Source (VCCS) provides:

$$
\left\{\begin{array}{l}
I_{\text {elec }}=G \cdot V_{\text {in }}-\frac{V_{\text {elec }}}{r_{\text {out }}} \\
G=\frac{1}{R_{1}} \\
r_{\text {out }}=\frac{R_{1} R_{2}\left(R_{2}+R_{3}\right)}{R_{3}^{2}-R_{2}^{2}}
\end{array}\right.
$$

where $G$ is the transconductance of the VCCS, and $r_{\text {out }}$ its output resistance. To allow accurate measurement, $r_{\text {out }}$ has to be huge compared to electrode impedance magnitude, therefore $R_{2}=R 3$ in this design. For this application we choose $R_{2}=R_{3}=100 \mathrm{k} \Omega$ with $0.01 \%$ tolerance ensuring a minimal output impedance of several $G \Omega$. Two values of $G$ $(1 \mathrm{mS}$ and $0.1 \mathrm{mS}$ ) have been used, corresponding respectively to $R_{1}=1 \mathrm{k} \Omega$ or $10 \mathrm{k} \Omega$. Current amplitude applied to the electrode varies from $1 \mu \mathrm{A}$ to $2 m A ; V_{\text {elec }}$ can be expected to cover the same range of variation. A low noise instrumentation amplifier (INA2128), controlled with different values of $R_{g}$, was added to provide gain for small voltage amplitudes and high input impedance (over $1 G \Omega$ ) in the recording loop.

Since the electrode impedance is defined as :

$$
Z_{\text {elec }}(s)=\frac{V_{\text {elec }}(s)}{I_{\text {elec }}(s)}
$$

where $s$ is the Laplace-domain variable, the measurement setup provide impedance evaluation with respect of :

$$
\left\{\begin{array}{l}
i_{\text {elec }}(t)=G \cdot V_{C h 1}(t) \\
v_{\text {elec }}(t)=\frac{V_{C h 2}(t)}{A_{v}}
\end{array}\right.
$$

where $A_{v}$ is the gain of the instrumentation amplifier, $V_{C h 1}$ and $V_{C h 2}$ are the recorded voltages on channels 1 and 2 (see Fig. 1(a)). This setup was used on a concentric bipolar electrode (NEX100, Phymep, France) used for DBS experimentation on rats. The electrode was placed in a Hank's Balanced Salt Solution (Thermo Scientific HyClone, SH3058801), which composition is similar to extra-cellular environment.

Signals injected through the electrode were charge balanced without DC, in order to prevent from electrochemical reactions [7]. Such signals with the same setup could be used in vivo as they ensure the integrity of living tissues surrounding the electrode.

\section{B. Measurement methods and results}

Typically, electrical neuronal stimulation induces physiological response by applying charged balanced constant current biphasic pulses. A first negative (cathodic) pulse initiates membrane depolarization of targeted cells ; then a positive (anodic) pulse allows to obtain an overall zero net charge at the end of a stimulation period.

Measurements with similar waveforms are shown in figure 1(b). Stimulation has been applied with current in the range from $50 \mu \mathrm{A}$ to $1 \mathrm{~mA}$ with a step of $50 \mu \mathrm{A}$. The duration of pulses has been set to $60 \mu \mathrm{s}$ as used for DBS. A delay of $940 \mu \mathrm{s}$ between cathodic and anodic pulses was used to observe interpulse voltage relaxation. Recording sampling frequency was set to $1.25 \mathrm{MHz}$ and measurement noise was reduced using an averaging method.

Measured voltages presented in figure 1(b) show in first approximation a integrator behavior during pulses. Nevertheless inter-pulse relaxation presents slow variations causing an asymetry in observed voltages. Such behavior cannot be explained by linear models based on serial and transfer resistances and double layer capacitance [9], but can be fitted using fractional order differential equations. Moreover, the voltage difference between two successive step responses tends 


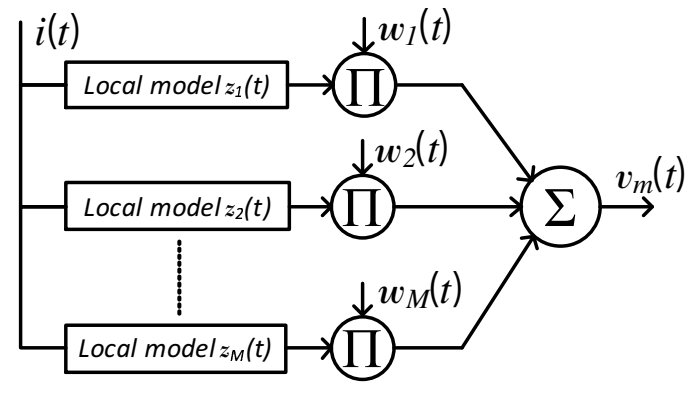

Fig. 2. Decoupled form multi-model

to decrease when the stimulation current increase, resulting in a decreasing impedance as observed in [10]. Currently, no measurement based computable model including fractional equation and current non-linearities can be found.

\section{EXTRACTION OF A NON LINEAR FRACTIONAL MODEL}

Taking into account the interface characterization described in section II, we used a multi-model approach to obtain a global non-linear model. The multi-model approach has been of interest since the Johansen and Foss publication [11]. To describe the non-linear behavior of a system, it uses a set of local linear models (or affine) of the system operation in various areas. The output of a local model is weighted by an activation function close to one if the operating point is near the center of the area associated to the local model, and decreasing towards zero when it moves away.

The standard form of a multi-model is:

$$
v_{m}(t)=\sum_{k=1}^{M} w_{k}(\varphi(t)) z_{k}(\varphi(t))
$$

where $\varphi(t)$ is an input-output observation vector at time $\mathrm{t}$, $w_{k}(\varphi(t))$ is the activation function of the local model $z_{k}, M$ the number of local models. Depending on the operating point, the activation function defines the contribution of the local model in the global model. It provides a gradual transition of this local model to the others neighboring local models. However, the following conditions must be imposed:

- the activation function is positive and less than 1 ,

$$
0 \leq w_{k}(\varphi(t)) \leq 1, \forall k, \forall t
$$

- the activation fuctions sum is equal to 1 at any point:

$$
\sum_{k=1}^{M} w_{k}(\varphi(t))=1, \forall t
$$

In this application the decoupled form multi-model is used as shown in figure 2. The function $\varphi(t)$ is the current applied to the interface $i(t)$. The activation functions $w_{k}(t)$ can be defined by:

$$
w_{k}(t)= \begin{cases}1-\lambda_{1, k}\left(I_{k}-i(t)\right) & \text { if } i(t) \in\left[I_{k-1}, I_{k}\right] \\ 1-\lambda_{2, k}\left(i(t)-I_{k}\right) & \text { if } i(t) \in\left[I_{k}, I_{k+1}\right] \\ 0 & \text { else }\end{cases}
$$

where $I_{k}$ denotes the operating point and where $\lambda_{j, k}$ is a coefficient that permits to meet equation (6). Other activation function shapes can be defined. Due to the diffusion phenomena that takes place in the interface [12], the local impedance models are defined using fractional transfer functions [13]:

$$
z_{k}(t)=\mathcal{L}^{-1}\left\{Z_{k}(s)\right\}
$$

where :

$$
Z_{k}(s)=K_{k}\left(\frac{\left(s / \omega_{b k}\right)^{\gamma_{k}}+1}{\left(s / \omega_{b k}\right)^{\gamma_{k}}}\right)
$$

The transfer function $Z_{k}$ is deduced from the shape of step responses from figure 1(b). Gain $K_{k}$, corner pulsation $\omega_{b k}$ and fractional order $\gamma_{k}$ are the result of an optimization problem that minimizes a quadratic criterion of time responses differences between model of equation (9) and measurements of figure 1(b). Examples of quantitative results are presented in table I.

\begin{tabular}{|l|c|c|c|c|c|}
\hline$I_{0}(\mu A)$ & $K_{k}(k \Omega)$ & $\omega_{b k}\left(\right.$ rad.s $\left.^{-1}\right)$ & $\gamma_{k}$ & $\lambda_{1, k}$ & $\lambda_{2, k}$ \\
\hline 50 & 477 & $5.78 \cdot 10^{4}$ & 0.60 & 0 & $2.2 \cdot 10^{3}$ \\
500 & 649 & $1.56 \cdot 10^{4}$ & 0.68 & $2.2 \cdot 10^{3}$ & $2 \cdot 10^{3}$ \\
1000 & 744 & $9.53 \cdot 10^{3}$ & 0.72 & $2 \cdot 10^{3}$ & 0 \\
\hline
\end{tabular}

TABLE I

EXAMPLES OF PARAMETERS OBTAINED FOR $Z_{k}(s)$ LOCAL MODELS AND CORRESPONDING $\lambda_{j, k}$

\section{MOdEL VALIDATION}

Step responses of local models given in table I where computed. Resulting voltage curves and corresponding measured responses are shown in figure 3(a). In order to simulate transfer functions similar to equation (9), differentiator of order $\gamma$ can be approximated by an integer order transfer function, stemming from a recursive distribution of poles and zeros as shown in [13]. Obtained results fit measurement for both step response and relaxation periods.

In order to validate the fractional multi-model a second experiment was conducted. A pseudo-random current sequence was injected through the electrode. This gaussian distributed sequence has a duration of $2 \mathrm{~ms}$, a sampling frequency of $1 M H z$, a root mean square of $308.3 \mu A$ and a maximal amplitude of $1 m A$ for the presented sequence. The measured voltage was then compared to simulation results using 3 different models :

- a fractional multi-model as described in section III ; 3 local models were used, which were extracted from $50 \mu A, 500 \mu A$ and $1 m A$ step responses to cover the global range of current variations,

- a single fractional model with the same form as equation (9), extracted from the $500 \mu \mathrm{A}$ step response,

- a linearized model as shown in [6] extracted from the $500 \mu A$ step response.

A $30 \mu \mathrm{s}$ zoomed view of obtained signal is shown in figure 3(b). Linearized model clearly presents a deviation from measured voltage. Both others fit with a smaller error the measured voltage. 


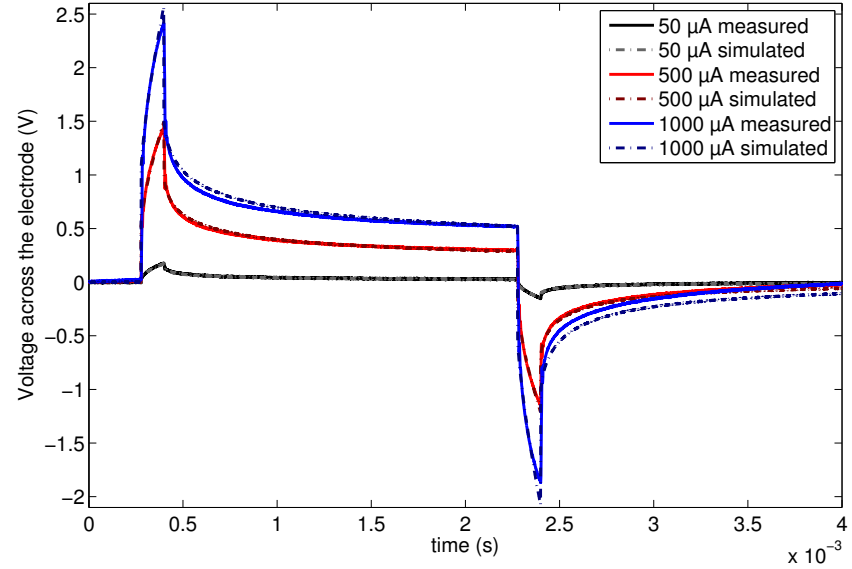

(a)

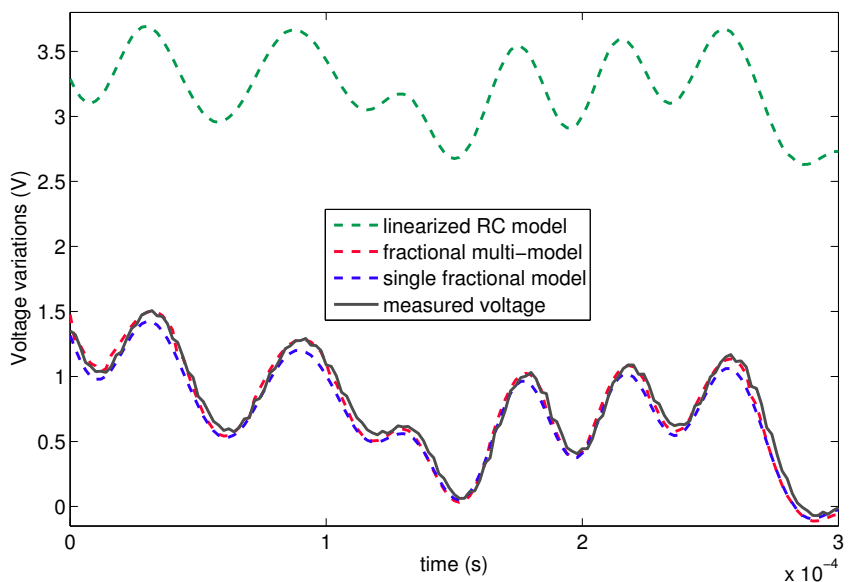

(b)

Fig. 3. 3(a) Comparison of measured step responses and simulated waveforms extracted from local models, 3(b) Comparison of voltage response to a pseudo-random current sequence obtained by measurement, fractional multi-model, single fractional model and linearized model.

In order to quantify models precision, the Normalized Root-Mean Square Error (NRMSE) between simulated and measured voltages was computed. For the linearized model response, the NRMSE is as high as $41.5 \%$, for the single fractional model, this error is $4.9 \%$ and for the multi-model NRMSE is $4.6 \%$. The multi-model shows a slightly better performance than the single model. However, the choice of the Gaussian distribution focuses on values around 0 to the detriment of high signal values, where the multi-model should be the most efficient. To complete this analysis, figure 3(b) provides a zoom on high values of voltage; on this interval, the NRMSE is $3.7 \%$ and $5.0 \%$ for the multi-model and the single fractional model respectively.

More generally the use of a high number of local models decreases the simulation error. Nevertheless all models are computed in parallel, so that increasing the number of local models increases the overall computation time.

\section{CONCLUSION}

We have presented a protocol for electrical characterization of the electrode-tissue interface, compatible with in vivo measurements. This technique is more suitable than the impedance spectroscopy to highlight the fractional and non linear nature of the interface. A classic model in the literature presents a ractional behavior as a constant phase element. Our original approach is based on a fractional multi-model, depending on the level of injected current and able to take into account the non-linear aspect. The identification method of local models from measured impulse responses is detailed. The multi-model is then evaluated with a random current stimulation; the results show an advantage of the multi-model on the simple fractional model.

This work is encouraging and allows to go further on the study of the non-linearity taking into account the physicochemical phenomena. The implementation of this model is based on recursive one order elements which allows to consider its use in a circuit simulator, using RC cells or a VHDLAMS description for CAD environment.

\section{REFERENCES}

[1] E. T. McAdams, A. Lackermeier, J. A. McLaughlin, D. Macken, J. Jossinet, "The linear and non-linear electrical properties of the electrode-electrolyte interface", Biosensors and Bioelectronics, vol. 10, issues 1-2, pp. 67-74, doi:10.1016/0956-5663(95)96795-Z, 1995.

[2] H. P. Schwan, "Linear and non-linear electrode polarization and biological materials", Annals of Biomedical Engineering, vol. 20, n. 3, pp. 269-288, doi:10.1007/BF02368531, 1992.

[3] S. H. Liu, "Fractal model for the AC response of a rough interface", Phys. Rev. Lett., vol. 55, issue 5, pp. 529-532, doi:10.1103/PhysRevLett.55.529, 1985.

[4] G. A. Ruiz, C. J. Felice, M. E. Valentinuzzi, "Non-linear response of electrode/electrolyte interface at high current density", Chaos, Solitons \& Fractals, vol. 25, n. 3, pp. 649-654, doi:10.1016/j.chaos.2004.11.029, 2005.

[5] E. A. Brown, J. D. Ross, R. A. Blum, Y. Nam, B. C. Wheeler, S. P. DeWeerth, "Stimulus Artefact Elimination in a Multi-Electrode System", IEEE Transactions on Biomedical Circuits and Systems, vol. 2, n. 1, pp. 10-21, doi:10.1109/TBCAS.2008.918285, 2008.

[6] S. K. Kelly, J. L. Wyatt, "A Power-Efficient Neural Tissue Stimulator With Energy Recovery", IEEE Transactions on Biomedical Circuits and Systems, vol. 5, n. 1, pp. 20-29, doi:10.1109/TBCAS.2010.2076384, 2011

[7] D. R. Merrill, M. Bikson,J. G.R. Jefferys, "Electrical stimulation of excitable tissue: design of efficacious and safe protocols", Journal of Neuroscience Methods, n. 141, pp. 171-198, doi:10.1016/j.jneumeth.2004.10.020, 2005.

[8] S. F. Cogan, "Neural Stimulation and Recording Electrodes", Annual Review of Biomedical Engineering, vol. 10, pp. 275-309, doi:10.1146/annurev.bioeng.10.061807.160518, 2008.

[9] X. F. Wei, W. M. Grill, "Impedance characteristics of deep brain stimulation electrodes in vitro and in vivo", Journal of Neural Engineering, vol. 6, n. 4, doi:10.1088/1741-2560/6/4/046008, 2009.

[10] S. F. Lempka, S. Miocinovic, M. D. Johnson, J. L. Vitej, C. C. McIntyre, "In vivo impedance spectroscopy of deep brain stimulation electrodes", Journal of Neural Engineering, vol. 6, n. 4, doi:10.1088/17412560/6/4/046001, 2009.

[11] T. A. Johansen, B. A. Foss, "Non-linear Local Model Representation For Adaptive Systems", Proceedings of International Conference on Intelligent Control and Instrumentation, vol. 2, pp. 677-682, doi:10.1109/SICICI.1992.637617, 1992.

[12] J. Sabatier, H. C. Nguyen, X. Moreau, A. Oustaloup, "Fractional behavior of partial differential equations whose coefficients are exponential functions of the space variable", Mathematical and Computer Modelling of Dynamical Systems, vol. 19, issue 5, doi: 10.1080/13873954.2013.766805, 2013.

[13] A. Oustaloup, F. Levron, B. Mathieu, F. M. Nanot, "Frequency-band Complex Noninteger Differentiator: Characterization and Synthesis", IEEE Transactions on Circuits and Systems-I: Fundamental Theory and Applications, vol. 47, n. 1, pp. 25-39, doi:10.1109/81.817385, 2000. 